\title{
A!
}

This is an electronic reprint of the original article.

This reprint may differ from the original in pagination and typographic detail.

Puska, M. J.; Mäkinen, S.; Manninen, Matti; Nieminen, Risto

\section{Screening of positrons in semiconductors and insulators}

Published in:

Physical Review B

DOI:

10.1103/PhysRevB.39.7666

Published: 15/04/1989

Document Version

Publisher's PDF, also known as Version of record

Please cite the original version:

Puska, M. J., Mäkinen, S., Manninen, M., \& Nieminen, R. (1989). Screening of positrons in semiconductors and insulators. Physical Review B, 39(11), 7666-7679. https://doi.org/10.1103/PhysRevB.39.7666

This material is protected by copyright and other intellectual property rights, and duplication or sale of all or part of any of the repository collections is not permitted, except that material may be duplicated by you for your research use or educational purposes in electronic or print form. You must obtain permission for any other use. Electronic or print copies may not be offered, whether for sale or otherwise to anyone who is not an authorised user. 


\title{
Screening of positrons in semiconductors and insulators
}

\author{
M. J. Puska* and S. Mäkinen \\ Department of Physics, University of Jyväskylä, SF-40100 Jyväskylä, Finland \\ M. Manninen ${ }^{\dagger}$ and R. M. Nieminen \\ Laboratory of Physics, Helsinki University of Technology, SF-02150 Espoo, Finland
}

(Received 8 June 1988)

\begin{abstract}
Theoretical models are presented for the enhancement of the electron density at a positron in a semiconductor or insulator host. The model better suited for typical semiconductors is based on the many-body theory for the screening of a positron in electron gas. The starting point of the model for insulators is the atomic polarizability. The common parameter in both models is the highfrequency dielectric constant. Moreover, the enhancement depends on the ambient electron density in the semiconductor model and on the unit-cell volume in the insulator model. With use of the models developed, positron lifetimes in perfect semiconductor and insulator crystals have been calculated. In the calculations, three-dimensional electron densities and electrostatic potentials are obtained by atomic superposition and the fully three-dimensional positron wave functions are solved by a relaxation method. The calculated positron lifetimes agree with the experimental ones within a few picoseconds. Moreover, we have used the model to predict lifetimes of positrons trapped by lattice defects such as vacancies and vacancy clusters.
\end{abstract}

\section{INTRODUCTION}

The electronic screening of a positron in condensed matter manifests itself in a very pronounced way: due to the electron pileup the observed positron lifetimes are much shorter than the ones predicted by the independent-particle model (IPM), which omits the electron-positron correlation effects. ${ }^{1}$ The screening of a positron in a metal is well understood on the basis of the many-body theory for a positive delocalized particle in a homogeneous electron gas. ${ }^{2}$ The enhancement of the electron density at the positron depends on the density of the unperturbed system. For instance, the enhancement factor for a high-density electron gas corresponding to the density parameter $r_{s}=2$ (atomic units) is about 4 and increases rapidly to the value of about 40 when the electron density decreases to $r_{s}=6$. The diverging behavior at the vanishing electron density is in fact due to the formation of a negative positronium ion ( $\mathrm{Ps}^{-}$) which guarantees a finite contact density also at this limit. The electron gas picture cannot be as such applied in the case of semiconductors or insulators because the polarizability or the screening efficiency of the electrons in the filled valence bands is, due to the lack of the low-energy excitations over the Fermi surface, less than the polarizability of the conduction electrons in metallic systems.

In this paper we introduce two simple models which describe the screening of positrons in semiconductors and insulators. The starting point of the first model is the above-mentioned theory for the positron in a homogeneous electron gas whereas the second model is based on the "atomic" polarizability of the constituents of the solid. It turns out that the former suits for the typical group-IV elemental and III-V and II-VI compound semiconductors while the latter works in the case of large- band-gap insulators. The borderline between semiconductors and insulators is, of course, not sharp and one should try to find a more general theory which covers both regions and which gives our two models at the appropriate limits. However, it has been found in this work that the two models overlap so that some of the host systems can be described equally well by both the models.

The two models not only give insight into the mechanisms of screening but also a quantitative description of its effect on the positron annihilation rate and the correlation energy. This is most useful for practical calculations for positron states in perfect and defected crystal lattices. The practical calculational schemes ${ }^{3}$ use the screening properties of positrons in the sense of the Kohn-Sham local-density approximation (LDA): the correlation energy and the annihilation rate at a given point in the host system depend only on the electron density at that point. Moreover, in the conventional scheme, which does not employ the two-component densityfunctional theory, ${ }^{4}$ these quantities are calculated for all states, i.e., including the localized ones, using the unperturbed host electron density (the zero positron density limit of the two-component theory).

The basis of the present work lies in the accurate measurements of positron lifetimes in bulk semiconductors and insulators. The trends seen in these provide guidelines for the models and the absolute values are the necessary test data to be reproduced in calculations. However, the extraction of the positron bulk lifetimes from the measured lifetime spectra is ambiguous due to the lifetime components connected with annihilation at defects in the samples, at sample surfaces, or in the positron source. For example, two values, 220 ps (Ref. 5) and $\sim 230 \mathrm{ps},{ }^{6,7}$ have been suggested for the bulk positron lifetime in GaAs. The difference is only 10 ps but is very 
important because it affects the interpretation of the experimental data in defect-containing samples and the crucial identification of defects present. Our calculations, free from adjustable parameters, will help in decomposing and interpreting the lifetime spectra.

The assignment of different lifetime components with different defects is an even more difficult task than the extraction of the positron bulk lifetimes as there can be several types of defects present simultaneously and in addition they can exist in different charge states. ${ }^{6,8,9}$ Theoretical predictions will help the analysis of the complicated data. ${ }^{10}$ Therefore we have applied the models in calculations of positron states in vacancies and divacancies in semiconductors and insulators.

\section{MODELS FOR POSITRON SCREENING}

\section{A. Sémiconductor model}

The screening of a positron by conduction electrons in metals can be described by the many-body calculations for a delocalized positron in a homogeneous electron gas. $^{2}$ The most important results from these calculations are the positron annihilation rate and the positron correlation energy. The former depends on the contact electron density $n(0)$, which is a function of the ambient electron density $n_{v}=3 / 4 \pi r_{s}^{3}$ (given in a.u.):

$$
\lambda_{v}=\pi r_{0}^{2} c n(0) \equiv \lambda_{0}\left(n_{v}\right)\left[1+\Delta g\left(n_{v}\right)\right],
$$

where

$$
\begin{aligned}
\lambda_{0}\left(n_{v}\right) & =\pi r_{0}^{2} c n_{v} \\
& =16 \pi n_{v}=12 / r_{s}^{3} \mathrm{~ns}^{-1}
\end{aligned}
$$

is the IPM result, in which $r_{0}$ is the classical electron radius and $c$ is the speed of light. Above, in Eq. (1) $\Delta g$ is the enhancement factor, which gives the relative pileup of the electron density at the positron. In the metallic density range, $2<r_{s}<6$, an accurate interpolation formula ${ }^{11}$ for the enhancement factor is

$$
\Delta g\left(n_{v}\right) \cong \frac{r_{s}^{3}+10}{6},
$$

or

$$
\lambda_{v}\left(n_{v}\right) \cong 2+134 n_{v} \mathrm{~ns}^{-1} .
$$

The latter form shows that the low-density limit for the annihilation rate is $2 \mathrm{~ns}^{-1}$, which corresponds to the spin-averaged lifetime of a Ps atom. ${ }^{12}$ The positron correlation energy is defined as the lowering of the total energy of the system due to the electron relaxation relative to the uniform distribution. It is also determined by many-body calculations ${ }^{2}$ for a positron in a homogeneous electron gas.

In semiconductors and insulators the existence of a band gap prevents the valence electrons from responding to external perturbations as effectively as the conduction electrons in metals. The screening is not perfect, which means that a stationary point charge $Z$ produces a longrange Coulomb potential with an asymptotic behavior proportional to $Z / \epsilon r$, where $\epsilon$ is the (static) dielectric constant of the medium. This means that the screening cloud of a point charge contains effectively $Z(1-1 / \epsilon)$ electrons. This fact is used in the following simple picture as a sum rule for the electron density screening the positron in a dielectric medium.

The electron density $n(r)$ screening a positive point charge obeys the cusp condition ${ }^{13}$ at the origin, where the point charge $Z$ is located, i.e.,

$$
\left.\frac{\partial n(r)}{\partial r}\right|_{r=0}=-2 Z n(0) \text {. }
$$

Let us assume that the screening charge decays exponentially as

$$
n(r)=n_{0}+C e^{-2 \alpha r},
$$

where $n_{0}$ is the unperturbed constant density. $C$ and $\alpha$ are parameters which are eliminated using the cusp condition and the sum rule

$$
\int_{0}^{\infty} C e^{-2 \alpha r} 4 \pi r^{2} d r=Z\left(1-\frac{1}{\epsilon}\right) .
$$

In the case of the light positron in the electronic system the cusp condition Eq. (5) has to be modified. At low electron densities the screening corresponds to the twobody (electron-positron) scattering, ${ }^{14}$ and it can be described by scattering of particles with charge $-e$ (electron charge) and mass $\mu=m / 2(m=$ electron mass) from a point charge $+e$ of infinite mass. As a result, the right-hand side of Eq. (5) has to be divided by two. Moreover, due to the light mass (strong recoil) of the positron it is more appropriate to use the high-frequency dielectric constant $\epsilon_{\infty}$. In other words, ions have no time to relax according to the instantaneous position of the positron. However, the static and high-frequency dielectric constants are not very different, so that both would give rather similar results for positron states and annihilation rates in semiconductors. With these substitutions the contact density calculated to the linear order in $n_{0}$ reads as

$$
n(0)=4 n_{0}+\frac{1}{8 \pi}\left(1-\frac{1}{\epsilon_{\infty}}\right) .
$$

Thereafter, the positron annihilation rate can be calculated from the contact electron density as

$$
\begin{aligned}
\lambda_{v} & =\pi r_{0}^{2} c n(0) \\
& =2\left[1-\frac{1}{\epsilon_{\infty}}\right]+201 n_{0} \mathrm{~ns}^{-1} .
\end{aligned}
$$

In order to make the result of this simple calculation agree with Eq. (4) at the metallic limit $\epsilon_{\infty}=\infty$ we have to substitute the numeric factor of 201 by 134 . As a matter of fact, Eq. (9) is the low electron density limit due to the approximations made in Eq. (8). Higher-order corrections to Eq. (9) bring it at finite densities $\left(r_{s} \sim 2 \ldots 6\right)$ closer to the result of Eq. (4), but then the annihilation rate is no longer linear in $n_{0}$. Therefore we prefer the 
above simple substitution and get the following parameter-free model for the positron annihilation rate in semiconductors:

$$
\begin{aligned}
\lambda_{v} & =2\left(1-\frac{1}{\epsilon_{\infty}}\right]+134 n_{0} \mathrm{~ns}^{-1} \\
& =\frac{12}{r_{s}^{3}}\left(1+\frac{\left(1-1 / \epsilon_{\infty}\right) r_{s}^{3}+10}{6}\right) \mathrm{ns}^{-1} .
\end{aligned}
$$

\section{B. Insulator model}

In insulators a more realistic approach than starting from the free-electron-gas model is based on the atomic polarizabilities of the constituents of the solid. The enhancement of the electron density at the positron is assumed in the lowest order to be proportional to the atomic polarizability $\alpha_{\text {at }}$, which in practical calculations can be estimated from the Clausius-Mossotti relation

$$
\alpha_{\mathrm{at}}=\frac{3 \Omega}{4 \pi} \frac{(\epsilon-1)}{(\epsilon+2)},
$$

where $\Omega$ is the unit-cell volume. The enhancement factor is now constant contrary to Eq. (10), which depends through the term $r_{s}^{3}$ on the local electron density. (However, as will be clearly demonstrated below, the unit-cell volume $\Omega$ and cube of the effective $r_{s}$ value affecting the positron in the solid are linearly related to each other.) The corresponding positron annihilation rate is

$$
\lambda_{v}=\frac{12}{r_{s}^{3}}\left[1+A+B \Omega \frac{\epsilon_{\infty}-1}{\epsilon_{\infty}+2}\right] \mathrm{ns}^{-1},
$$

where $A$ and $B$ are parameters which can be determined by fitting the calculated positron lifetimes to the experimental ones. The parameter $A$ will make the enhancement factor always larger than 1.5 , which is the accepted value for tightly bound core electrons. ${ }^{15}$ We have found (see below) that the following values reproduce best the existing experimental data:

$$
A=0.684, \quad B=0.0240 \text {. }
$$

Due to the two parameters introduced, the insulator model can be considered rather as an interpolation scheme, contrary to the semiconductor model Eq. (10), which has no adjustable parameters. The insulator model is applied in this work mainly to solids with covalent bonds or to solids with partial ionic-bond character. The application to positron states in rare-gas solids, which previously have been studied extensively, would require a more careful treatment ${ }^{16}$ of the correlation effects due to atomic polarization, because $\epsilon_{\infty}$ is very close to unity for these solids.

\section{Previous models}

Brandt and Reinheimer ${ }^{17}$ studied in a pioneering work the screening of a positive point charge in semiconductors and insulators. They used the Penn model, ${ }^{18}$ in which the host is mimicked by a homogeneous electron gas with an energy gap $\left(E_{g}\right)$ in the parabolic density of states. They fitted $E_{g}$ so that the dielectric function calculated in the model approaches at the zero-frequency limit the experimental static dielectric constant. The width of the valence band is simply the Fermi energy $\left(E_{F}\right)$ in the free-electron model with the average valence electron density of the semiconductor. They found that the positron annihilation rate can be expressed as

$$
\lambda_{v}=\frac{12}{r_{s}^{3}}\left[1+f\left(r_{s}, \varepsilon_{g}\right) \frac{r_{s}^{3}+10}{6}\right] \mathrm{ns}^{-1},
$$

where $f\left(r_{s}, \varepsilon_{g}\right)$ is a factor describing the reduction of electron enhancement in semiconductors relative to metals for which $f=1$ gives Eq. (4). Above, $\varepsilon_{g}$ is the "gap parameter" related to the widths of the band gap and the valence band as

$$
\varepsilon_{g}=\frac{E_{g}}{E_{F}} \text {. }
$$

Brandt and Reinheimer estimated [within the BohmPines random-phase approximation (RPA)] the reduction factor $f$ for a set of $r_{s}$ and $\varepsilon_{g}$ values. A reasonable fit to the data reads ${ }^{19}$

$$
f\left(r_{s}, \varepsilon_{g}\right)=1-\frac{0.37 \varepsilon_{g}}{1+0.18 r_{s}} .
$$

When inserted in Eq. (14) this does not give the present form in Eq. (10) and the resulting enhancement factor is generally too small. However, if $\varepsilon_{g}$ is used as a free parameter, a single value, $\varepsilon_{g}=0.2$, reproduces well the experimental bulk lifetimes for several semiconductors. ${ }^{3}$

Chiba et $a .^{20}$ have developed a theoretical model for describing the positron correlation with tightly bound electrons such as those in insulators. They derived an enhancement factor which depends on the binding energy and on the electron momentum of the core level. For practical calculations they made the Thomas-Fermi approximation in which the electron binding energy and the local Fermi momentum are expressed in terms of the local electron density. It follows that the enhancement factor then is a function of the electron density only. The weak point of this approximation is, as will be seen below, that the observed variations in the enhancement factor are so large that they cannot be described as arising only from the density variations.

\section{Correlation potential and the practical calculations}

When applying Eqs. (10) and (12) for calculations of positron states and annihilation rates the correlation energy used in the LDA potential should be consistent with them. A scaling argument ${ }^{1}$ asserts for the correlation energy

$$
E_{\text {corr }} \sim\left(\lambda-\lambda_{0}\right)^{1 / 3},
$$

where $\lambda$ is the actual annihilation rate and $\lambda_{0}$ is the IPM result Eq. (2). The correlation energy has been calculat$\mathrm{ed}^{2}$ for the case of a homogeneous electron gas and a practical interpolation formula ${ }^{4}$ is available. This can be used for semiconductor and insulator systems described 
by Eqs. (10) and (12) by scaling the electron-gas result $E_{\text {corr }}^{\mathrm{EG}}$ as

$$
E_{\mathrm{corr}}=E_{\mathrm{corr}}^{\mathrm{EG}}\left(\frac{\Delta g}{\Delta g_{\mathrm{EG}}}\right)^{1 / 3},
$$

where $\Delta g_{E G}$ and $\Delta g$ are the enhancement factors for the electron gas and for the present models, respectively.

In actual calculations in which the discrete lattice structure with the inhomogeneous electron density has been included the above formulas have to be used in the sense of the local approximation. In the conventional scheme $^{3}$ for calculating positron states the electron structure undisturbed by the presence of the positron is used. The potential affecting the positron at point $\mathbf{r}$ in the host is then obtained as

$$
V_{+}(\mathbf{r})=V_{\text {Coulomb }}(\mathbf{r})+E_{\text {corr }}(n(\mathbf{r})),
$$

where $V_{\text {Coulomb }}$ is the Coulomb potential rising from the host charge distribution and $n(\mathbf{r})$ is the host electron density. Next, the corresponding positron wave function $\Psi_{+}$is solved. The total positron annihilation rate $\lambda$ and the positron lifetime $\tau$ are calculated then also in the local approximation as

$$
\lambda=1 / \tau=\int d \mathbf{r}\left|\Psi_{+}\right|^{2}\left[\lambda_{v}\left(n_{v}(\mathbf{r})\right)+\lambda_{c}\left(n_{c}(\mathbf{r})\right)\right] .
$$

Above, the annihilation rates with valence $\left(n_{v}\right)$ and core $\left(n_{c}\right)$ electrons are taken into account separately. Equation (4), (10), or (12) should be used for $\lambda_{v}$, whereas the annihilation rate with core electrons can be calculated starting from the IPM result and using a constant enhancement factor of $\gamma_{c}=1.5$, i.e.

$$
\lambda_{c}\left(n_{c}\right)=16 \pi \gamma_{c} n_{c} \mathrm{~ns}^{-1} \text {. }
$$

In this work we have performed the calculations using the practical method introduced by Puska and Nieminen. ${ }^{10}$ The host electron density and the Coulomb potential are constructed by superimposing free atoms, solved by a computer program using the local-density approximation $^{21}$ for the electron exchange and correlation. The fully three-dimensional Schrödinger equation is solved for the positron wave function and eigenenergy by a numerical relaxation method. The mesh points, which are used to describe the potential, electron density, and the positron wave function in the crystal form an orthorombic Bravais lattice. Translation and point symmetries are used to reduce the integration volume as much as possible. The calculation scheme is known to be powerful for describing positron states in perfect metal lattices, in point defects, and on metal surfaces. ${ }^{10} \mathrm{Re}-$ cently, it has been successfully used for semiconductors $\mathrm{Si}$ and $\mathrm{GaAs}^{22}$

\section{POSITRONS IN PERFECT LATTICES}

\section{A. Results}

The present calculations rely on the fact that the electron density in a semiconductor can quickly and reasonably well be obtained by the superposition of free-atom

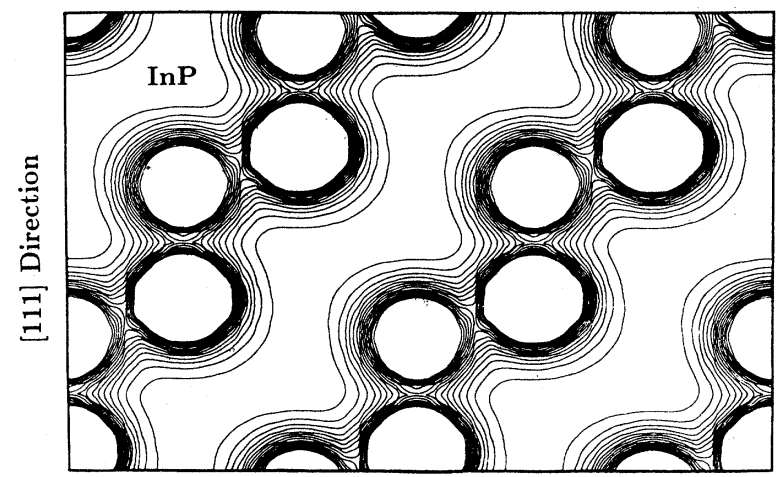

$[11 \overline{2}]$ Direction

FIG. 1. Total electron density in InP. Atomic superposition is used. The value for the contour nearest to the interstitial region is $0.01 a_{0}^{-3}$. The values increase monotonically towards the ion cores with the spacing of $0.005 a_{0}^{-3}$.

electron densities. In this work we even use spherical atomic densities. The resulting electron density in the zinc-blende lattice structure is shown in Fig. 1 in the case of InP. The superimposed electron density does not show the pileup at the bonds between the neighboring atoms. The charge transfer towards ionicity cannot be taken properly into account either. However, when compared with self-consistent electron structure calculations, ${ }^{23}$ the superposition describes surprisingly well the electron density at the interstitial regions and its rise close to the atom chains. In fact, these are the most important aspects determining the positron state and the annihilation rate because the positron has a strong tendency to seek out into open interstitial regions. This tendency is clearly seen in Fig. 2, which shows the delocalized positron wave function in the ideal InP lattice. The superposition of atomic densities may be most questionable for the most

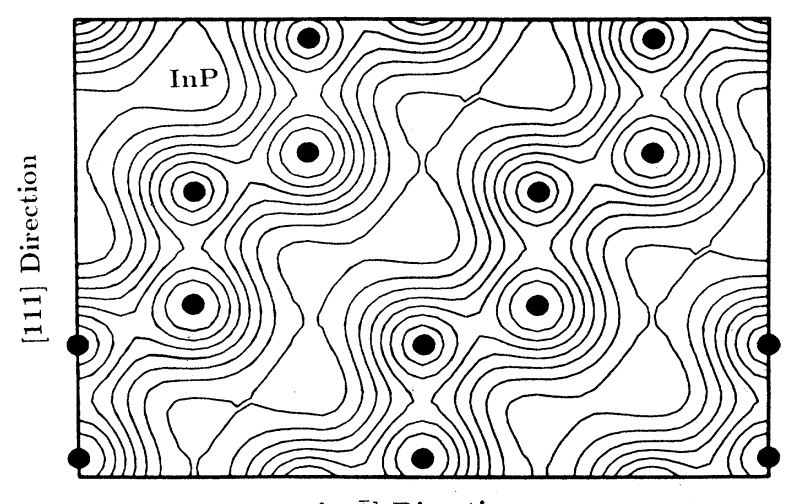

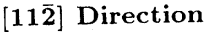

FIG. 2. Delocalized positron wave function in perfect InP. The ion positions are denoted by solid circles. The lower of the nearest neighbors in the (111) direction is In and the upper one $P$. The wave function is at maximum in the interstitial regions between the ion chains and vanishes at the nuclei. The contour spacing is $\frac{1}{8}$ of the maximum value. 
ionic compounds treated in this work. For this kind of cases self-consistent electronic structures could give more reliable results, but their calculation is enormously more complicated than the atomic superposition.

The lattice constants and high-frequency dielectric functions used in the present calculations for semiconductors and insulators are listed in Table I. Moreover, several experimental values for the positron lifetime in the perfect lattices are given and we have chosen an "average" value $\tau_{\text {expt }}^{*}$ for the experiment-theory comparisons. The positron annihilation characteristics calculated according to the semiconductor model [Eqs. (10) and (18)] are collected in Table II for several perfect semiconductor and insulator lattices. The corresponding data arising from the insulator model [Eqs. (12), (13), and (18)] are shown in Table III. The calculated positron bulk lifetimes are in good agreement with the experimental values $\tau_{\text {expt }}^{*}$ given in Table I, if $\mathrm{C}, \mathrm{GaN}, \mathrm{MgO}, \mathrm{SiC}$, and $\mathrm{GaP}$ are treated as insulators and the rest of the hosts as semiconductors. The good agreement between calculated and measured results is demonstrated also in Figs. 3(a) and 3(b), which show the bulk lifetimes as a function of the unit-cell volume of the lattice. This agreement gives credence to the simple models for the positron screening used and to the interpretation of experimental lifetime spectra.

In the insulator model the dependence on the dielectric constant $\epsilon_{\infty}$ is much stronger than in the case of the semiconductor model. This leads to a larger scatter in the bulk lifetimes when they are plotted as a function of the unit-cell volume in Fig. 3(a). For the typical insulators the agreement between the theoretical and experimental lifetimes is not as good as for semiconductors. This may be partly due to the fact that the experimental data in Fig. 3 are taken from several sources. Systematic experimental work is called for to further clarify positron annihilation in insulators.

The core annihilation rates for the hosts studied are generally rather small, typically $\sim 0.3 \mathrm{~ns}^{-1}$; for $\mathrm{C}, \mathrm{BP}$, and $\mathrm{Si}$ as small as $\sim 0.1 \mathrm{~ns}^{-1}$. Only in the case of insulators $\mathrm{GaN}$ and $\mathrm{MgO}$ do they make a rather large contribution to the total annihilation rates. The core annihilation

TABLE I. Data for semiconductors and insulators. In the calculations the zinc-blende or diamond structures are used, except in the case of $\mathrm{MgO}$, which has the sodium chloride structure. The lattice constants $a$ (Ref. 24), high-frequency dielectric constants $\epsilon_{\infty}$ (Ref. 25), and experimental positron bulk lifetimes $\tau_{\text {expt }}$ are given. The lattice parameter for $\mathrm{GaN}$ in the zinc-blende structure has been calculated by assuming the density of the actual wurtzite lattice structure. $\tau_{\text {expt }}^{*}$ is the "average" experimental positron bulk lifetime used in experiment-theory comparisons in this work.

\begin{tabular}{|c|c|c|c|c|c|}
\hline Host & $\begin{array}{c}a \\
\text { (a.u.) } \\
\end{array}$ & $\epsilon_{\infty}$ & & $\begin{array}{l}\tau_{\text {expt }} \\
\text { (ps) }\end{array}$ & $\begin{array}{c}\tau_{\text {expt }}^{*} \\
(\mathrm{ps})\end{array}$ \\
\hline $\mathrm{Si}$ & 10.26 & 12.0 & & $218,^{\mathrm{a}} 219, \mathrm{~b} 222^{\mathrm{c}}$ & 220 \\
\hline $\mathrm{Ge}$ & 10.69 & 16.0 & & $228,^{\mathrm{c}} 230^{\mathrm{d}}$ & 229 \\
\hline AlP & 10.30 & 7.6 & & & \\
\hline AlAs & 10.61 & 8.2 & & & \\
\hline AlSb & 11.61 & 10.2 & & & \\
\hline GaP & 10.30 & 9.1 & & $223,{ }^{\mathrm{e}} 225^{\mathrm{f}}$ & 224 \\
\hline GaAs & 10.69 & 10.9 & $220,{ }^{\mathrm{g}} 230,{ }^{\mathrm{h}}$ & h $231,{ }^{c} 232,{ }^{\mathrm{i}} 235^{\mathrm{j}}$ & 232 \\
\hline $\mathrm{GaSb}$ & 11.57 & 14.4 & & $247,^{f} 260,{ }^{k} 260^{1}$ & 260 \\
\hline InP & 11.09 & 9.6 & $235,{ }^{\mathrm{f}} 2$ & $242,^{\mathrm{c}} 244,^{\mathrm{m}} 247^{\mathrm{k}}$ & 244 \\
\hline InAs & 11.43 & 12.3 & & $247,^{\mathrm{f}} 257^{\mathrm{c}}$ & 257 \\
\hline $\mathrm{InSb}$ & 12.26 & 15.7 & & $258,^{\mathrm{f}} 280,{ }^{\mathrm{k}} 282^{\mathrm{n}}$ & 280 \\
\hline $\mathrm{CdTe}$ & 12.26 & 7.2 & & $289,^{\circ} 291^{f}$ & \\
\hline $\mathrm{HgTe}$ & 12.22 & 14.0 & & $274^{\circ}$ & \\
\hline $\mathrm{BeO}$ & 7.20 & 3.0 & & & \\
\hline BP & 8.60 & 8.2 & & & \\
\hline $\mathrm{C}$ & 6.72 & 5.66 & & $115^{f}$ & 115 \\
\hline $\mathrm{GaN}$ & 8.47 & 5.4 & & $180^{k}$ & 180 \\
\hline $\mathrm{MgO}$ & 7.96 & 3.0 & & $166^{p}$ & 166 \\
\hline $\mathrm{SiC}$ & 8.21 & 6.6 & & $157^{\mathrm{f}}$ & 157 \\
\hline $\begin{array}{l}{ }^{\mathrm{a}} \text { Reference } 26 . \\
\text { beference } 27 . \\
{ }^{\mathrm{b}} \text { Refere } 7 . \\
{ }^{\mathrm{c}} \text { Reference } 7 . \\
\text { d Reference } 28 . \\
{ }^{\mathrm{e}} \text { Reference } 29 . \\
{ }^{\mathrm{f}} \text { Reference } 30 . \\
\text { g Reference } 5 . \\
{ }^{\mathrm{h}} \text { Reference } 8 .\end{array}$ & & & $\begin{array}{l}{ }^{\mathrm{i}} \text { Reference } 6 . \\
{ }^{\mathrm{j}} \text { Reference } 31 . \\
{ }^{\mathrm{k}} \text { Reference } 32 . \\
{ }^{1} \text { Reference } 33 . \\
{ }^{\mathrm{m}} \text { Reference } 34 . \\
{ }^{\mathrm{n}} \text { Reference } 35 . \\
{ }^{\circ} \text { Reference } 36 . \\
{ }^{\mathrm{p}} \text { Reference } 37 .\end{array}$ & & \\
\hline
\end{tabular}


TABLE II. Positron annihilation characteristics in perfect lattices calculated using the semiconductor model [Eq. (7)] for screening. $\lambda_{v}$ and $\lambda_{c}$ are the annihilation rates due to the valence and core electrons, respectively. $\tau$ is the positron bulk lifetime, $r_{s}^{\text {eff }}$ corresponds to the average electron density affecting the positron, and $\Delta g^{\text {eff }}$ is the enhancement factor defined in Eq. (19). The hosts which are well described by the semiconductor model are indicated by an asterisk.

\begin{tabular}{lccccc}
\hline Host & $\begin{array}{c}\lambda_{v} \\
\left(\mathrm{~ns}^{-1}\right)\end{array}$ & $\begin{array}{c}\lambda_{c} \\
\left(\mathrm{~ns}^{-1}\right)\end{array}$ & $\begin{array}{c}\tau \\
(\mathrm{ps})\end{array}$ & $\begin{array}{c}r_{s}^{\text {eff }} \\
(\mathrm{a} . \mathrm{u} .)\end{array}$ & $\Delta g^{\text {eff }}$ \\
\hline${ }^{*} \mathrm{Si}$ & 4.471 & 0.103 & 219 & 2.298 & 3.520 \\
${ }^{*} \mathrm{Ge}$ & 4.129 & 0.301 & 226 & 2.421 & 3.884 \\
& & & & & \\
${ }^{*} \mathrm{AlP}$ & 4.274 & 0.120 & 228 & 2.328 & 3.492 \\
${ }^{*} \mathrm{AlAs}$ & 4.072 & 0.183 & 235 & 2.400 & 3.688 \\
${ }^{*} \mathrm{AlSb}$ & 3.545 & 0.170 & 269 & 2.639 & 4.429 \\
GaP & 4.271 & 0.315 & 218 & 2.342 & 3.572 \\
${ }^{*} \mathrm{GaAs}$ & 4.021 & 0.340 & 229 & 2.439 & 3.864 \\
${ }^{*} \mathrm{GaSb}$ & 3.600 & 0.287 & 257 & 2.640 & 4.521 \\
${ }^{*} \mathrm{InP}$ & 3.733 & 0.339 & 246 & 2.545 & 4.127 \\
${ }^{*} \mathrm{InAs}$ & 3.592 & 0.346 & 254 & 2.632 & 4.458 \\
${ }^{*} \mathrm{InSb}$ & 3.291 & 0.283 & 280 & 2.825 & 5.186 \\
& & & & & \\
$\mathrm{BeO}$ & 8.834 & 0.208 & 111 & 1.622 & 2.141 \\
$\mathrm{BP}$ & 6.497 & 0.123 & 151 & 1.890 & 2.654 \\
$\mathrm{C}$ & 11.88 & 0.145 & 83 & 1.462 & 2.096 \\
$\mathrm{GaN}$ & 6.326 & 0.767 & 141 & 1.896 & 2.592 \\
$\mathrm{MgO}$ & 7.586 & 0.790 & 119 & 1.723 & 2.235 \\
$\mathrm{SiC}$ & 7.283 & 0.198 & 134 & 1.789 & 2.477 \\
\hline \hline
\end{tabular}

TABLE III. Positron annihilation characteristics in perfect lattices calculated using the insulator model [Eq. (9)] for screening. $\lambda_{v}$ and $\lambda_{c}$ are the annihilation rates due to the valence and core electrons, respectively. $\tau$ is the positron bulk lifetime, $r_{s}^{\text {eff }}$ corresponds to the average electron density affecting the positron, and $\Delta g$ is the enhancement factor defined in Eq. (19). The hosts which are well described by the insulator model are indicated by an asterisk.

\begin{tabular}{lccccc}
\hline Host & $\begin{array}{c}\lambda_{v} \\
\left(\mathrm{~ns}^{-1}\right)\end{array}$ & $\begin{array}{c}\lambda_{c} \\
\left(\mathrm{~ns}^{-1}\right)\end{array}$ & $\begin{array}{c}\tau \\
(\mathrm{ps})\end{array}$ & $\begin{array}{c}r_{s}^{\text {eff }} \\
(\text { a.u. })\end{array}$ & $\Delta g$ \\
\hline $\mathrm{Si}$ & 4.405 & 0.128 & 221 & 2.259 & 3.231 \\
$\mathrm{Ge}$ & 4.328 & 0.395 & 212 & 2.361 & 3.745 \\
& & & & & \\
$\mathrm{AlP}$ & 3.959 & 0.147 & 244 & 2.286 & 2.941 \\
$\mathrm{AlAs}$ & 3.898 & 0.232 & 242 & 2.350 & 3.216 \\
$\mathrm{AlSb}$ & 3.740 & 0.240 & 251 & 2.560 & 4.230 \\
${ }^{*} \mathrm{GaP}$ & 4.069 & 0.388 & 224 & 2.292 & 3.080 \\
$\mathrm{GaAs}$ & 4.034 & 0.441 & 223 & 2.375 & 3.503 \\
$\mathrm{GaSb}$ & 3.977 & 0.413 & 228 & 2.548 & 4.482 \\
$\mathrm{InP}$ & 3.772 & 0.443 & 237 & 2.467 & 3.719 \\
InAs & 3.848 & 0.481 & 231 & 2.537 & 4.234 \\
InSb & 3.846 & 0.443 & 233 & 2.697 & 5.287 \\
& & & & & \\
${ }^{*} \mathrm{BeO}$ & 8.834 & 0.209 & 111 & 1.426 & 1.133 \\
${ }^{*} \mathrm{BP}$ & 6.583 & 0.134 & 149 & 1.768 & 2.032 \\
*C & 8.617 & 0.143 & 114 & 1.461 & 1.239 \\
*GaN & 4.947 & 0.821 & 173 & 1.887 & 1.769 \\
* MgO & 5.190 & 0.791 & 167 & 1.743 & 1.290 \\
${ }^{*} \mathrm{SiC}$ & 5.907 & 0.211 & 163 & 1.778 & 1.765 \\
\hline \hline
\end{tabular}
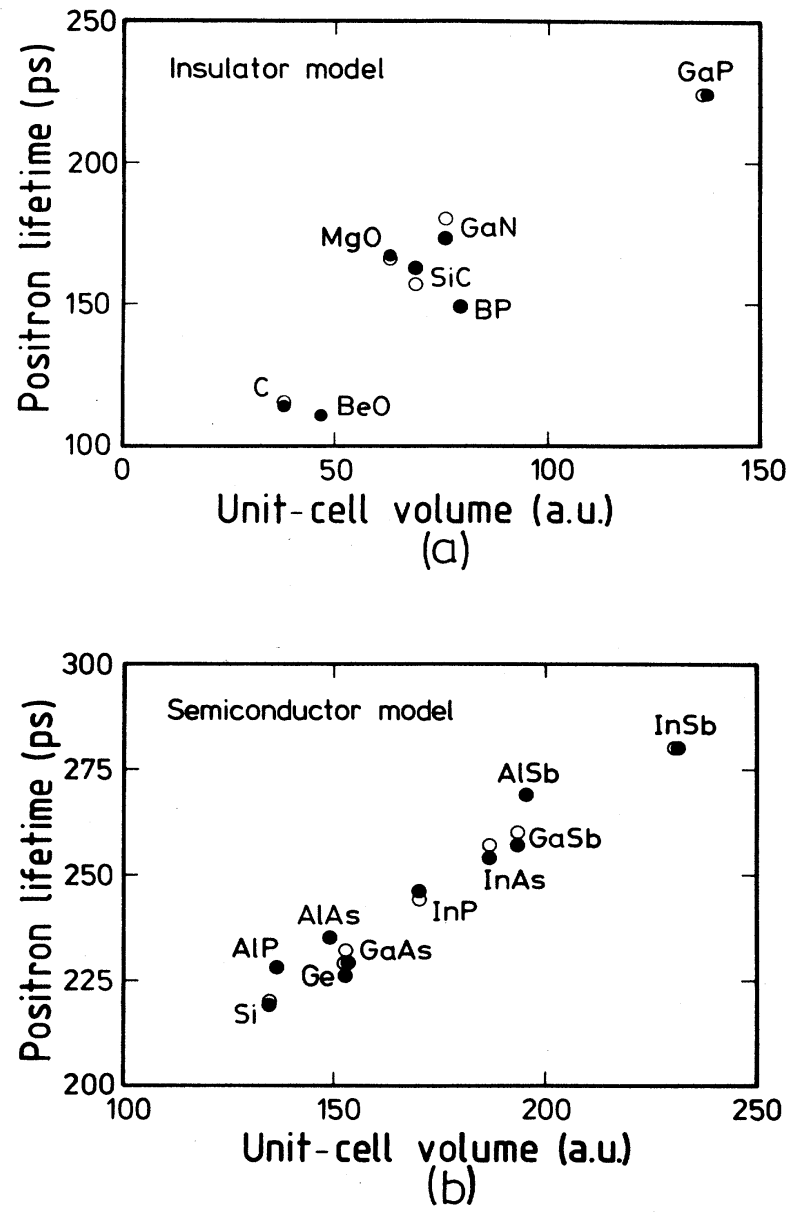

FIG. 3. The positron lifetimes in perfect insulator and semiconductor lattices as a function of the unit-cell volume. The theoretical values (solid circles) are obtained in (a) by using the insulator model [Eq. (12)] and in (b) by using the semiconductor model [Eq. (10)]. The experimental values (open circles) are the $\tau_{\text {expt }}^{*}$ values given in Table I.

rate depends on the core enhancement factor. For example, in the case of semiconductors, if the IPM [with the value of 1.0 for the enhancement factor instead of 1.5 (Ref. 15)] is used the positron lifetimes increase typically by 5 ps. Thus the choice of the core enhancement factor may cause small systematic errors in the calculated positron lifetimes.

\section{B. Discussion}

In order to achieve criteria for which of the two models should be chosen in a specific case we analyze the positron annihilation in bulk semiconductors and insulators in more detail. We define a density parameter $r_{s}^{\text {eff }}$ which corresponds to the effective valence electron density with respect to the positron annihilation. This means that $r_{s}^{\text {eff }}$ inserted in Eq. (10) or (12) gives the valence annihilation rates in Tables II and III, i.e., rates which are obtained by integrating over the lattice volume using the threedimensional positron and electron densities. Another im- 


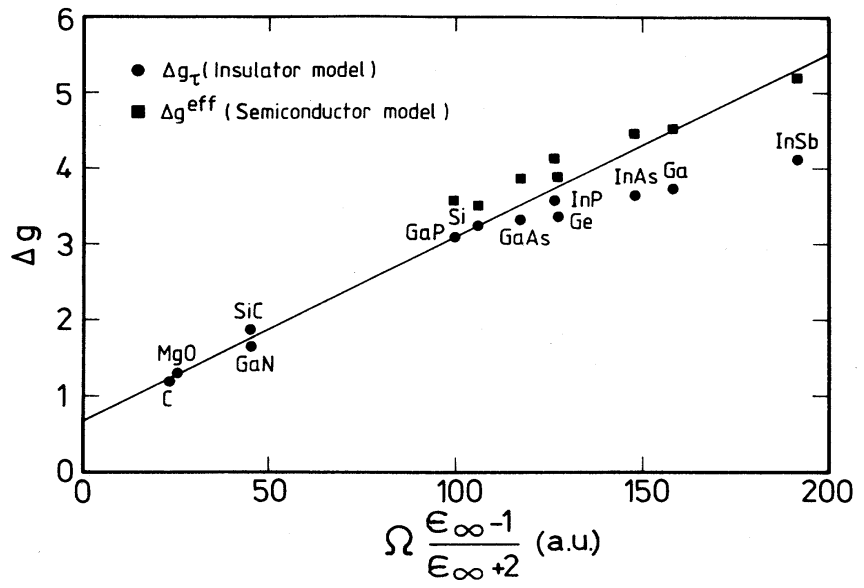

FIG. 4. The enhancement factors $\Delta g_{\tau}$ (solid circles) reproducing the experimental positron lifetimes $\tau_{\text {expt }}^{*}$ in the insulator model. For the definition, see Eq. (23). The abscissa is proportional to the atomic polarizability in Eq. (11). The line shown is the linear least-squares fit to the data for $\mathrm{C}, \mathrm{GaN}, \mathrm{MgO}, \mathrm{SiC}$, and GaP. The slope and the vertical axis intercept give the parameters $A$ and $B$ of the insulator model Eq. (12). For comparison, the enhancement factors $\Delta g^{\text {eff }}$ (solid squares) calculated by Eq. (22) in the semiconductor model are also shown.

portant parameter is the enhancement $\Delta g$ which for semiconductor (SC) and insulator (IN) models is, according to Eqs. (1), (10), and (12),

$$
\begin{aligned}
& \Delta g^{\mathrm{eff}}=\frac{\left(1-1 / \epsilon_{\infty}\right)\left(r_{s}^{\text {eff }}\right)^{3}+10}{6}, \mathrm{SC}, \\
& \Delta g=A+B \Omega \frac{\epsilon_{\infty}-1}{\epsilon_{\infty}+2}, \mathrm{IN} .
\end{aligned}
$$

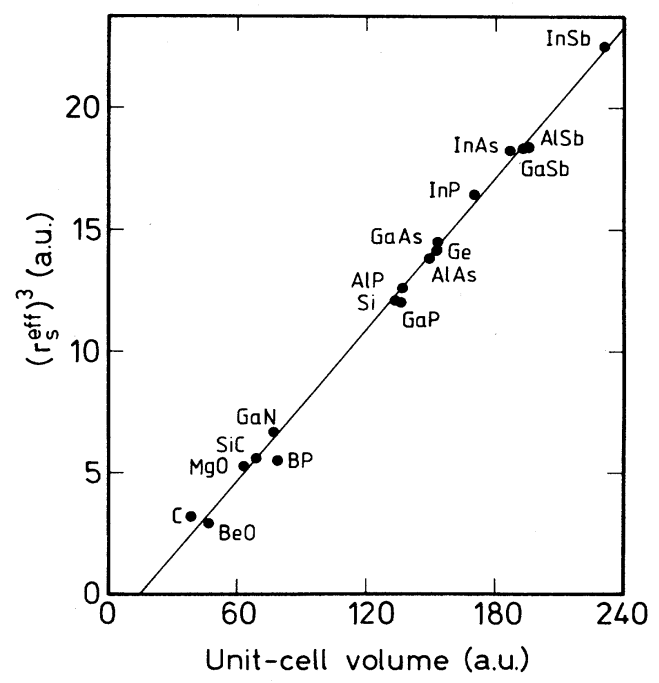

FIG. 5. The cube of the effective electron density parameter seen by the positron as a function of the unit-cell volume. For the definition, see text. The line shown is the linear leastsquares fit [Eq. (24)] to the data.

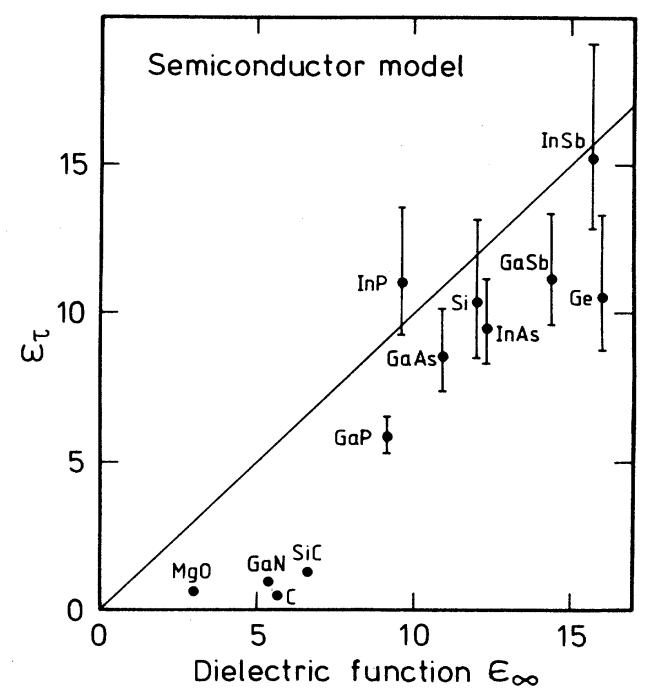

FIG. 6. The dielectric constants $\epsilon_{\tau}$ calculated from the positron bulk lifetimes $\tau_{\text {expt }}^{*}$. The values correspond to the semiconductor model in Eq. (25). The abscissa is the measured highfrequency dielectric constant. The line shown corresponds to $\epsilon_{\tau}=\epsilon_{\infty}$. The error bars correspond to an uncertainty of $\pm 2 \mathrm{ps}$ in the positron lifetime.

When the semiconductor model is applied in the real three-dimensional calculations the enhancement at a given point depends on the local electron density. In the upper part of Eq. (22) the effective electron density $r_{s}^{\text {eff }}$ is used and therefore the enhancement factor obtained is also an effective one, $\Delta g$ eff. On the other hand, in the insulator model the enhancement is a density-independent constant as indicated also in the notation.

Figure 4 illustrates the determination of the parameters $A$ and $B$ appearing in the formulas for the annihila-

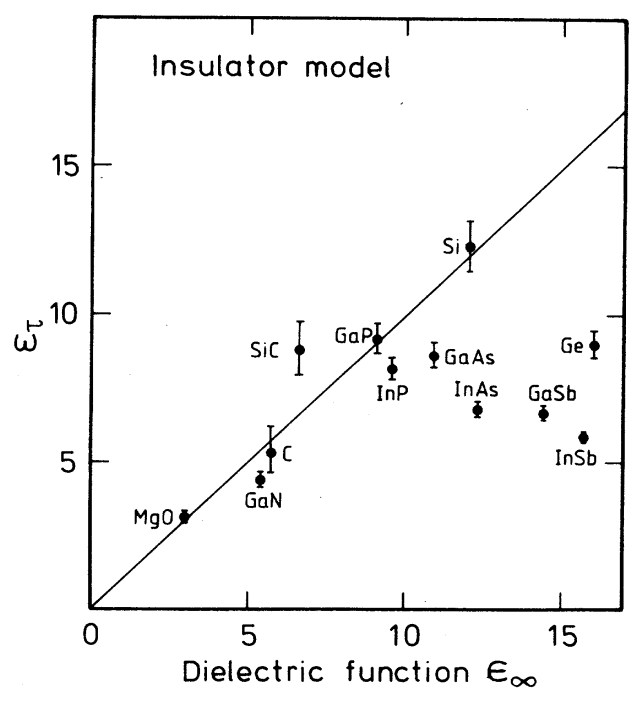

FIG. 7. Same as Fig. 6 but for the insulator model in Eq. (25). 
tion rate [Eq. (12)] and enhancement $\Delta g$ [Eq. (22)] in the insulator model. The enhancement factors $\Delta g_{\tau}$, which reproduce the experimental positron lifetimes $\tau_{\text {expt }}^{*}$ given in Table $I$, are plotted as a function of the atomic polarizability Eq. (11). As a matter of fact, this determination should be done in a self-consistent way, because $\Delta g_{\tau}$ 's are calculated as

$$
\Delta g_{\tau}=\frac{\left(r_{s}^{\mathrm{eff}}\right)^{3}}{12}\left[\frac{1}{\tau_{\mathrm{expt}}^{*}}-\lambda_{c}\right]-1
$$

where $r_{s}^{\text {eff }}$ (given in a.u.) and $\lambda_{c}\left(\right.$ in $\mathrm{ns}^{-1}$ ) are the theoretical results depending on the values of $A$ and $B$ ( $\tau_{\text {expt }}^{*}$ is given in ns). However, this dependence comes through the correlation potential [Eq. (18)] and is very weak. Therefore only one improvement iteration is needed. The points for $\mathrm{C}, \mathrm{GaN}, \mathrm{MgO}, \mathrm{SiC}, \mathrm{GaP}$, and $\mathrm{Si}$ fall nicely on the same straight line determining the parameters $A$ and $B$ as in Eq. (13). The semiconductors to the right of $\mathrm{Si}$ fall below this line, indicating that the linear dependence assumed in the insulator model is no longer adequate. In order to fully assess the model for insulators more data is clearly needed, especially to fill the gap between SiC and GaP.

From Tables II and III we can see that Si can be described equally well in both models, i.e., the ranges of validity of the models overlap. The fact that the models for insulators and semiconductors seem to join rather smoothly means that the borderline between these models is not sharp. In practice, one may choose the model to be used by calculating the value of $\Omega\left[\left(\epsilon_{\infty}-1\right) /\left(\epsilon_{\infty}+2\right)\right]$ for the system in question and comparing it to the borderline of the models where it is around 100-110 a.u.

The values of $r_{s}^{\text {eff }}$ and $\Delta g$ for the "borderline" case Si clarify the differences between the two models. $\Delta g^{\text {eff's }}$ calculated in the semiconductor model for several hosts are added as squares in Fig. 4 , in which the straight line gives the insulator model result, and the circles denote the constant enhancements $\Delta g_{\tau}$ reproducing the experimental positron lifetimes. The high electron density regions near the ion cores contribute to the positron annihilation more in the insulator model than in the semiconductor model, because the enhancement is constant in the former whereas in the latter model the enhancement decreases when the electron density increases. Therefore the $r_{s}^{\text {eff' }} s$ are smaller in the insulator model. In the annihilation rate this difference is compensated by a smaller enhancement in the insulator model. Therefore both models give similar rates for $\mathrm{Si}$. However, to the right of $\mathrm{Si}$, e.g., in the cases of $\mathrm{GaAs}$ and $\mathrm{Ge}$, the compensation in enhancement should be larger than given by the insulator model (the $\Delta g_{\tau}$ 's fall below the straight line). As a consequence, the insulator model gives, e.g., for GaAs and Ge, a shorter positron lifetime than the semiconductor model.

The cubes $\left(r_{s}^{\text {eff }}\right)^{3}$ are plotted in Fig. 5 as a function of the unit-cell volume. They fall in a more or less straight line

$$
\left(r_{s}^{\mathrm{eff}}\right)^{3} \simeq 0.104 \Omega-1.58
$$

This means that the "open volume" for the positron in the interstitial regions of the host is directly proportional to the unit-cell volume. The $r_{s}^{\text {eff }}$ values are remarkably larger than the values resulting if the valence electrons were uniformly distributed over the whole unit-cell volume. For example, $r_{s}^{\text {eff }}=2.30$ for $\mathrm{Si}$ whereas the conventionally calculated $r_{s}=2.01$. The difference reflects the fact that in semiconductors positrons reside in the interstitial regions whereas the valence electron density resides in bonds between the neighboring atoms. This tendency would be even clearer if the electron density were self-consistent instead of the superposition of free atoms. For comparison, for the fcc metal Al the atomic superposition calculation ${ }^{10}$ gives $r_{s}^{\text {eff }}=2.10$, which is very close to the conventional $r_{s}=2.07$.

The dependence of the annihilation rate on the dielectric constant $\epsilon_{\infty}$ becomes weaker when $\epsilon_{\infty}$ increases in the insulator model [Eq. (12)] and this tendency continues even more clearly in the semiconductor model [Eq. (10)]. In order to demonstrate the importance of the $\epsilon_{\infty}$ dependence in the annihilation rate we have solved Eqs. (10) and (12) for the dielectric constant:

$$
\begin{aligned}
& \epsilon_{\tau}=\frac{1}{1-\frac{1}{2}\left(1 / \tau_{\text {expt }}^{*}-\lambda_{c}^{\text {theor }}-134 n_{\text {theor }}^{\text {eff }}\right)}, \mathrm{SC}, \\
& \epsilon_{\tau}=\frac{B \Omega+2\left[\left(\frac{1}{\tau_{\text {expt }}^{*}}-\lambda_{c}^{\text {theor }}\right) \frac{\left(r_{s, \text { theor }}^{\text {eff }}\right)^{3}}{12}-1-A\right]}{B \Omega-\left[\left(\frac{1}{\tau_{\text {expt }}^{*}}-\lambda_{c}^{\text {theor }}\right) \frac{\left(r_{s, \text { theor }}^{\text {eff }}\right)^{3}}{12}-1-A\right]}, \mathrm{IN} \mathrm{.}
\end{aligned}
$$

Thereafter, as indicated already above, we use the calculated core electron annihilation rates $\lambda_{c}^{\text {theor }}$ (given in $n s^{-1}$ ), the calculated effective electron densities $n_{\text {theor }}^{\text {eff }}$ (in a.u.) or $r_{s, \text { theor }}^{\text {eff }}$ ' (in a.u.), and the experimental positron

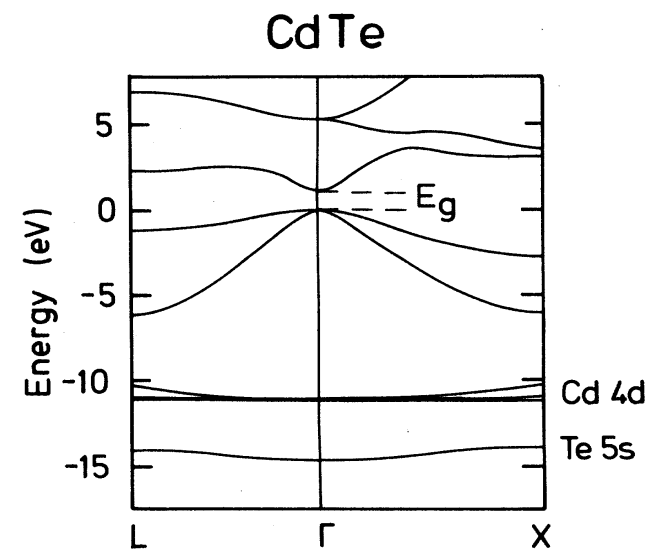

FIG. 8. Self-consistent electron energy-band structure for CdTe. The calculation is performed by the LMTO-ASA method. Relativistic effects, except the spin-orbit coupling, are included (scalar relativistic bands). The zero of the energy scale is the top of the valence band. 
lifetimes $\tau_{\text {expt }}^{*}$ (in ns) and obtain a dielectric constant $\epsilon_{\tau}$ corresponding to the measured positron lifetime. All the values needed are given in Tables I, II, and III. In fact, this is a very good test for the models proposed, because the theoretical results for $\lambda_{c}^{\text {theor }}$ and $r_{s, \text { theor }}^{\text {eff }}$ in the given model depend only weakly on the dielectric constant $\epsilon_{\infty}$ through the correlation potential [Eq. (18); note the cube-root dependence]. Moreover, a rigorous test would be to use the $\epsilon_{\tau}$ value obtained from Eq. (25) in a new positron state calculation and thereby to make an iteration towards "self-consistency." The results calculated using the data in Tables I, II, and III are shown in Figs. 6 and 7 for the semiconductor and insulator models, respectively. The error bars correspond to an uncertainty of \pm 2 ps in the positron lifetime. The semiconductor model in Fig. 6 gives reasonable results when $\epsilon_{\infty}$ is greater than $\sim 9$, but fails drastically for the typical insulators with small $\epsilon_{\infty}$ values. The error bars are rather large especially for the high- $\epsilon_{\infty}$ semiconductors. The insulator model works very well when $\epsilon_{\infty}$ is less than $\sim 9$, but it is interesting to note that it can predict dielectric constants also for InP, $\mathrm{GaAs}$, and $\mathrm{Si}$. For higher $\epsilon_{\infty}$ the $\epsilon_{\tau}$ values seem to decline. Thus the regions of validity of the two models overlap also in this sense. The strong correlations seen in Figs. 6 and 7 imply that the positron lifetime measurements could be used in a sense to determine the highfrequency dielectric constants for semiconductors and insulators.

In the case of GaAs, there is a debate concerning the bulk lifetime value. Dannefaer et $a l . .^{5}$ have strongly suggested that it should be $\sim 220$ ps, which is shorter than the value of $\sim 230 \mathrm{ps}$ found by other experimentalists (see Table I). The value of $230 \mathrm{ps}$ for GaAs as well as the experimental bulk lifetimes shown in Fig. 3 are obtained from measurements for $p$-type and semi-insulating semiconductors for which one-component lifetime spectra are obtained after subtraction of small source terms. This single component is then interpreted as the positron bulk lifetime. On the other hand, the result of Dannefaer et al. is obtained by fitting the trapping model to the decompositions of experimental spectra, i.e., not directly as an observed lifetime component. Dannefaer has been able to decompose the measured spectra by gathering a relatively large number of counts in the lifetime spectra. Theoretically, the lifetime of 220 ps can be reproduced by using the metallic limit $\left(\epsilon_{\infty}=\infty\right)$ in Eq. (10). However, in order to be consistent one should then observe, e.g., for $\mathrm{Si}$, a bulk lifetime substantially lower than 220 ps, which is the value accepted also by Dannefaer. On the other hand, if the insulator model described above were valid for GaAs, the predicted positron bulk lifetime would be, according to Table III, 223 ps. But, because GaAs is on the right-hand side of $\mathrm{Si}$ on the polarizability scale (Fig. 4), we prefer to use the semiconductor model, which works already for $\mathrm{Si}$.

Previously, ${ }^{19}$ positron bulk lifetimes in semiconductors have been calculated with the linear muffin-tin orbitals (LMTO) method within the atomic-spheres approximation (LMTO-ASA). ${ }^{38}$ The electron structures in these calculations are self-consistent although the actual threedimensional geometry is not exactly taken into account due to the ASA. In these calculations the form (11) by Brandt and Reinheimer ${ }^{17}$ is used for the annihilation rate, and the value of $\varepsilon_{g}=0.2$ is chosen in order to reproduce the experimental bulk lifetimes in average. The lifetime results agree well with the present ones showing that the electronic structure can well be approximated for this purpose by the superposition of free atoms.

\section{II-VI compound semiconductors}

A prominent feature in the electron band structure of II-VI compound semiconductors is the narrow $d$ bands at rather high energies. For example, Fig. 8 shows the band structure for $\mathrm{CdTe}$, which has been calculated using the LMTO-ASA method. The Cd $4 d$ bands are in the heteropolar gap, slightly separated from the valence band. The Te $5 s$ bands lie below the Cd $4 d$ bands, but their width is still considerable. The problem in calculating positron annihilation rates in these materials is the description of the enhancement for the $d$ bands. It should be larger than the enhancement for the tightly bound core but less than that for the more delocalized valence electrons. We have used the same scheme as in the context ${ }^{10}$ of the transition metals, i.e., the enhancement factor of the $d$ elec-

TABLE IV. Positron annihilation characteristics in perfect CdTe and in $\mathrm{HgTe}$ lattices. The calculations are performed using the semiconductor model [Eq. (7)] for positron annihilation with valence electrons, which include $\mathrm{Cd} 5 s, \mathrm{Hg} 6 s$, and $\mathrm{Te} 5 s$ and $5 p$ electrons in the atomic superposition. Cd $4 d$ and $\mathrm{Hg} 5 d$ electrons are treated with a constant enhancement factor $\gamma_{d}$. For $\gamma_{d}$ either the value of 1.5 corresponding to the tightly bound core electrons or the values appropriate for $d$ electrons in $\mathrm{Ag}(1.95)$ and $\mathrm{Au}$ (2.35) metals are used. $\lambda_{v}, \lambda_{c}$, and $\lambda_{d}$ are the annihilation rates due to the valence, core, and $d$ electrons, respectively. $\tau$ is the positron bulk lifetime, $r_{s}^{\text {eff }}$ corresponds to the average electron density affecting the positron, and $\Delta g$ eff is the enhancement factor defined in Eq. (19).

\begin{tabular}{lllccccr}
\hline \hline Host & $\gamma_{d}$ & $\begin{array}{c}\lambda_{v} \\
\left(\mathrm{~ns}^{-1}\right)\end{array}$ & $\begin{array}{c}\lambda_{c} \\
\left(\mathrm{~ns}^{-1}\right)\end{array}$ & $\begin{array}{c}\lambda_{d} \\
\left(\mathrm{~ns}^{-1}\right)\end{array}$ & $\begin{array}{c}\tau \\
(\mathrm{ps})\end{array}$ & $\begin{array}{c}r_{s}^{\text {eff }} \\
(\mathrm{a} . \mathrm{u} .)\end{array}$ & $\Delta g^{\text {eff }}$ \\
\hline CdTe & 1.5 & 3.054 & 0.141 & 0.234 & 292 & 2.885 & 5.114 \\
& 1.95 & & & 0.304 & 286 & & \\
$\mathrm{HgTe}$ & 1.5 & 3.206 & 0.150 & 0.281 & 275 & 2.873 & 5.338 \\
& 2.35 & & & 0.440 & 263 & & \\
\hline \hline
\end{tabular}


trons is a density-independent constant (this should be larger than 1.5 used for the core). For the valence electrons the above semiconductor model has been used. We have included the outermost $s$ electrons of the column-VI component to the valence electrons. This is justified, as in the solid they form a band of more delocalized electrons than the electrons in the highest $d$ band of the column-II component. For example, according to our LMTO-ASA calculations about $96 \%$ of the Cd $4 d$ electrons in $\mathrm{CdTe}$ are localized in the atomic spheres (radius 3.0156 a.u.) surrounding the Cd nuclei, whereas the localization of the $\mathrm{Te} 5 \mathrm{~s}$ electrons in the similar spheres around the Te nuclei is about $75 \%$.

The positron annihilation characteristics calculated for perfect CdTe and HgTe lattices are given in Table IV. Two limits are considered. The use of the core enhancement factor of 1.5 also for uppermost $d$ electrons gives the upper limit for the positron lifetime. The lower limit is obtained by using the $d$-enhancement factors determined in the case of nearby $\mathrm{Ag}$ and $\mathrm{Au}$ metals for $\mathrm{Cd}$ and $\mathrm{Hg}$, respectively. These enhancement factors are certainly too high, because in the present cases the localization of the $d$ electrons is stronger due to the larger atomic numbers and the larger distances between the metal atoms. For CdTe the two lifetime estimates are rather close to each other and to the values obtained experimentally (Table I). The scatter is much larger for $\mathrm{HgTe}$, where the higher limit is nearer the experimental estimate (Table I). As a matter of fact, the experimental values are somewhat uncertain because the extraction of positron bulk lifetimes from the many-component spectra has been difficult. ${ }^{36}$ However, the present calculations confirm the simple picture that from these two semiconductors, which have nearly the same lattice constants, $\mathrm{HgTe}$ should have the smaller positron lifetime, because the $\mathrm{Hg}$ atom is larger than the $\mathrm{Cd}$ atom. The calculated $r_{s}^{\text {eff }}$ and $\Delta g^{\text {eff }}$ values for $\mathrm{CdTe}$ and $\mathrm{HgTe}$ are also shown in Table IV. They lie within the values typical for semiconductors and would be situated near InSb in Figs. 4 and 5. To conclude, the semiconductor model presented above

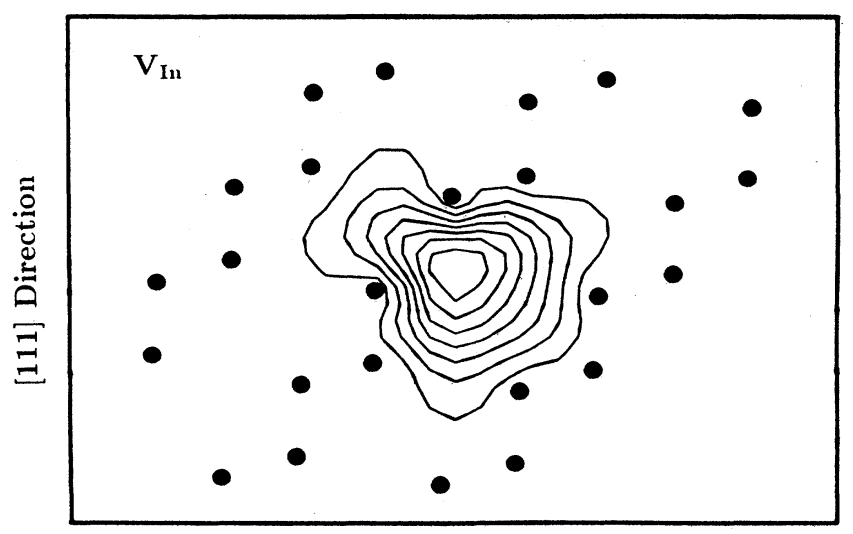

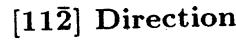

FIG. 9. Localized positron wave function in the In vacancy in InP. The ion positions are denoted by solid circles. The contour spacing is $\frac{1}{8}$ of the maximum value.

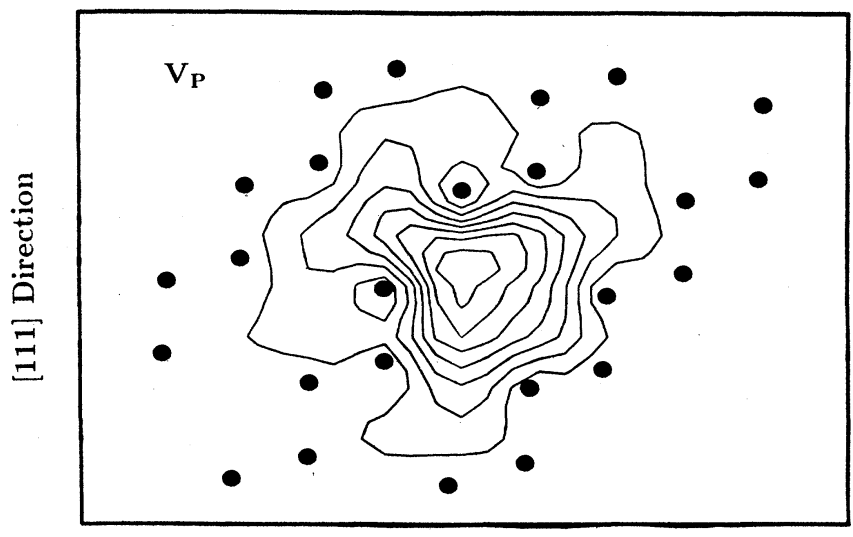

[11̄̄] Direction

FIG. 10. Localized positron wave function in the $P$ vacancy in InP. The ion positions are denoted by solid circles. The contour spacing is $\frac{1}{8}$ of the maximum value.

can be used with minor modifications to include $d$ electron enhancement descriptions of positron states and annihilation rates in II-VI compound semiconductors as well.

\section{POSITRONS TRAPPED BY VACANCIES AND DIVACANCIES}

\section{A. Results}

The insulator and semiconductor models presented form a basis for calculations of positron states and annihilation rates also in defected lattices. Here we show results for ideal vacancies and nearest-neighbor divacancies in insulators and semiconductors, i.e., the lattice relaxation around the defect is not taken into account. Moreover, the atomic superposition used does not allow description of differences in the electron structures between the different charge states of the defect.

The wave function of a positron trapped by the two

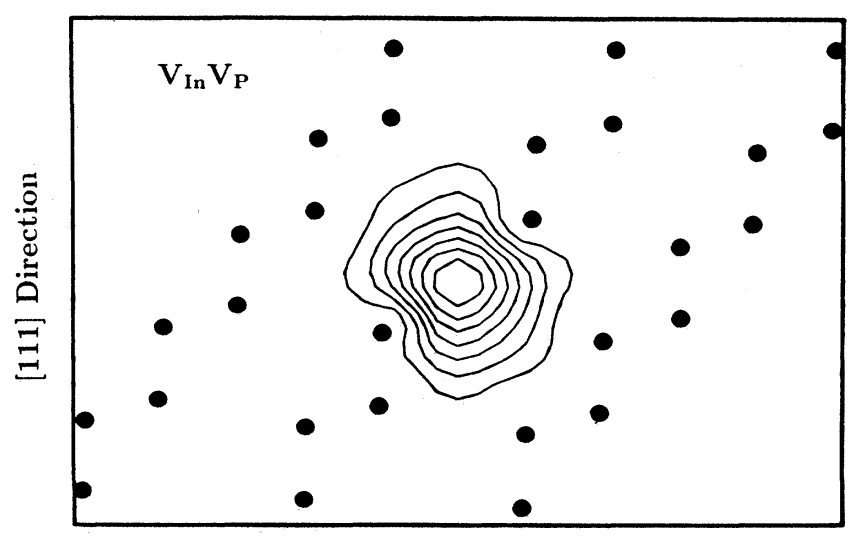

[112] Direction

FIG. 11. Localized positron wave function in the nearestneighbor divacancy in InP. The ion positions are denoted by solid circles. The contour spacing is $\frac{1}{8}$ of the maximum value. 
kinds of vacancies and the nearest-neighbor divacancy in InP are shown in Figs. 9-11. The localization in the vacancies is weaker than in typical metal vacancies. Especially, in the smaller $P$ vacancy (Fig. 10) the wave function has a tendency to flow over into the open interstitial regions. It is seen that the open volume corresponding to the divacancy is needed to maintain localization similar to that at metal vacancies. The difference between the two kinds of vacancies with respect to the positron response is much clearer in the insulators studied than in the III-V semiconductors. In $\mathrm{GaN}, \mathrm{SiC}$, and $\mathrm{MgO}$, we find that $N, C$, and $O$ vacancies cannot bind positrons at all. This is interesting, because, for example in the case of $\mathrm{MgO}$, one expects that the $\mathrm{O}$ vacancies are positively

TABLE V. Properties of positrons trapped at vacancies and nearest-neighbor divacancies in insulators and semiconductors. For $\mathrm{C}, \mathrm{SiC}, \mathrm{GaN}, \mathrm{MgO}, \mathrm{BeO}, \mathrm{BP}$, and $\mathrm{GaP}$ the calculations are performed using the insulator model [Eq. (9)] whereas in the other cases the semiconductor model [Eq. (7)] is used. $\mathrm{Cd} 4 d$ and $\mathrm{Hg} 5 d$ electrons are treated as tightly bound core electrons with the enhancement factor $\gamma_{d}=1.5 . \lambda_{v}$ and $\lambda_{c}$ are the annihilation rates due to the valence and core electrons, respectively. $\tau$ is the positron lifetime, $r_{s}^{\text {eff }}$ corresponds to the average electron density affecting the positron, and $\Delta g$ is the enhancement factor defined in Eq. (19). $E_{b}$ is the positron binding energy in the defect.

\begin{tabular}{|c|c|c|c|c|c|c|c|}
\hline Host & Defect & $\begin{array}{c}\lambda_{v} \\
\left(\mathrm{~ns}^{-1}\right)\end{array}$ & $\begin{array}{c}\lambda_{c} \\
\left(\mathrm{~ns}^{-1}\right)\end{array}$ & $\begin{array}{c}\tau \\
\text { (ps) }\end{array}$ & $\begin{array}{c}r_{s}^{\text {eff }} \\
\text { (a.u.) }\end{array}$ & $\Delta g$ & $\begin{array}{c}E_{b} \\
(\mathrm{eV}) \\
\end{array}$ \\
\hline \multirow[t]{2}{*}{$\mathrm{Si}$} & $\mathrm{Si}$ & 3.859 & 0.053 & 256 & 2.509 & 4.080 & 0.42 \\
\hline & Divac. & 3.204 & 0.029 & 309 & 2.858 & 5.233 & 1.29 \\
\hline \multirow[t]{2}{*}{$\mathrm{Ge}$} & $\mathrm{Ge}$ & 3.624 & 0.173 & 263 & 2.635 & 4.526 & 0.36 \\
\hline & Divac. & 3.059 & 0.102 & 316 & 3.001 & 5.891 & 1.10 \\
\hline \multirow[t]{3}{*}{ AlP } & $\mathrm{Al}$ & 3.727 & 0.050 & 265 & 2.524 & 3.993 & 0.59 \\
\hline & $\mathbf{P}$ & 3.749 & 0.077 & 261 & 2.515 & 3.968 & 0.16 \\
\hline & Divac. & 3.101 & 0.034 & 319 & 2.862 & 5.061 & 1.19 \\
\hline \multirow[t]{3}{*}{ AlAs } & Al & 3.593 & 0.102 & 271 & 2.592 & 4.215 & 0.50 \\
\hline & As & 3.542 & 0.103 & 274 & 2.617 & 4.288 & 0.22 \\
\hline & Divac. & 2.257 & 0.023 & 439 & 3.997 & 11.01 & 1.57 \\
\hline \multirow[t]{3}{*}{$\mathrm{AlSb}$} & $\mathrm{Al}$ & 3.240 & 0.114 & 298 & 2.814 & 5.015 & 0.26 \\
\hline & $\mathrm{Sb}$ & 3.050 & 0.084 & 319 & 2.950 & 5.526 & 0.35 \\
\hline & Divac. & 2.170 & 0.026 & 455 & 4.438 & 14.80 & 1.26 \\
\hline \multirow[t]{3}{*}{$\mathrm{GaP}$} & $\mathrm{Ga}$ & 3.558 & 0.233 & 264 & 2.396 & 3.080 & 0.08 \\
\hline & $\mathbf{P}$ & & & & & & $<0$ \\
\hline & Divac. & 2.949 & 0.212 & 316 & 2.551 & 3.080 & 0.27 \\
\hline \multirow[t]{3}{*}{ GaAs } & $\mathrm{Ga}$ & 3.600 & 0.173 & 265 & 2.618 & 4.382 & 0.44 \\
\hline & As & 3.496 & 0.229 & 268 & 2.671 & 4.551 & 0.24 \\
\hline & Divac. & 2.994 & 0.118 & 321 & 3.006 & 5.779 & 1.03 \\
\hline \multirow[t]{3}{*}{$\mathrm{GaSb}$} & $\mathbf{G a}$ & 3.318 & 0.171 & 287 & 2.800 & 5.072 & 0.21 \\
\hline & $\mathrm{Sb}$ & 3.087 & 0.169 & 307 & 2.967 & 5.716 & 0.38 \\
\hline & Divac. & 2.760 & 0.100 & 350 & 3.290 & 7.188 & 0.93 \\
\hline \multirow[t]{3}{*}{ InP } & In & 3.263 & 0.129 & 295 & 2.791 & 4.914 & 0.60 \\
\hline & $\mathbf{P}$ & 3.380 & 0.283 & 273 & 2.721 & 4.675 & 0.06 \\
\hline & Divac. & 2.829 & 0.110 & 340 & 3.136 & 6.273 & 1.05 \\
\hline \multirow[t]{3}{*}{ InAs } & In & 3.194 & 0.155 & 299 & 2.868 & 5.279 & 0.49 \\
\hline & As & 3.241 & 0.265 & 285 & 2.835 & 5.157 & 0.11 \\
\hline & Divac. & 2.769 & 0.117 & 347 & 3.251 & 6.928 & 0.98 \\
\hline \multirow[t]{3}{*}{ InSb } & In & 3.019 & 0.152 & 315 & 3.034 & 6.024 & 0.30 \\
\hline & $\mathrm{Sb}$ & 2.915 & 0.194 & 322 & 3.131 & 6.455 & 0.26 \\
\hline & Divac. & 2.608 & 0.099 & 369 & 3.517 & 8.456 & 0.88 \\
\hline \multirow[t]{3}{*}{$\mathrm{CdTe}$} & $\mathrm{Cd}$ & 2.893 & 0.226 & 321 & 3.012 & 5.588 & 0.11 \\
\hline & $\mathrm{Te}$ & 2.668 & 0.286 & 339 & 3.234 & 6.521 & 0.21 \\
\hline & Divac. & 2.444 & 0.157 & 384 & 3.540 & 8.033 & 0.60 \\
\hline
\end{tabular}


TABLE V. (Continued).

\begin{tabular}{|c|c|c|c|c|c|c|c|}
\hline Host & Defect & $\begin{array}{c}\lambda_{v} \\
\left(\mathrm{~ns}^{-1}\right)\end{array}$ & $\begin{array}{c}\lambda_{c} \\
\left(\mathrm{~ns}^{-1}\right)\end{array}$ & $\begin{array}{c}\tau \\
(\mathrm{ps})\end{array}$ & $\begin{array}{c}r_{s}^{\text {eff }} \\
\text { (a.u.) }\end{array}$ & $\Delta g$ & $\begin{array}{c}E_{b} \\
(\mathrm{eV})\end{array}$ \\
\hline \multirow[t]{3}{*}{$\mathrm{HgTe}$} & $\mathrm{Hg}$ & 3.033 & 0.252 & 304 & 3.008 & 5.880 & 0.16 \\
\hline & $\mathrm{Te}$ & 2.820 & 0.355 & 315 & 3.215 & 6.809 & 0.22 \\
\hline & Divac. & 2.578 & 0.184 & 362 & 3.542 & 8.542 & 0.66 \\
\hline \multirow[t]{3}{*}{$\mathrm{BeO}$} & $\mathrm{Be}$ & 7.719 & 0.067 & 128 & 1.491 & 1.133 & 0.73 \\
\hline & O & & & & & & $<0$ \\
\hline & Divac. & 6.156 & 0.075 & 160 & 1.608 & 1.133 & 1.46 \\
\hline \multirow[t]{3}{*}{ BP } & B & & & & & & $<0$ \\
\hline & $\mathbf{P}$ & 5.367 & 0.069 & 184 & 1.893 & 2.032 & 0.39 \\
\hline & Divac. & 4.712 & 0.055 & 210 & 1.977 & 2.032 & 0.89 \\
\hline \multirow[t]{2}{*}{ C } & C & 6.796 & 0.074 & 146 & 1.581 & 1.239 & 0.60 \\
\hline & Divac. & 4.799 & 0.048 & 206 & 1.776 & 1.239 & 2.17 \\
\hline \multirow[t]{2}{*}{$\mathrm{GaN}$} & Ga & 3.457 & 0.211 & 273 & 2.126 & 1.769 & $<0$ \\
\hline & Divac. & 2.666 & 0.205 & 348 & 2.319 & 1.769 & 2.00 \\
\hline \multirow[t]{2}{*}{$\mathrm{MgO}$} & $\mathrm{Mg}$ & 3.571 & 0.182 & 266 & 1.974 & 1.290 & $<0$ \\
\hline & Divac. & 3.622 & 0.136 & 266 & 1.965 & 1.290 & 2.46 \\
\hline \multirow[t]{3}{*}{$\mathrm{SiC}$} & $\mathrm{Si}$ & 3.961 & 0.056 & 249 & 2.031 & 1.765 & 1.44 \\
\hline & C & & & & & & $<0$ \\
\hline & Divac. & 3.026 & 0.046 & 326 & 2.222 & 1.765 & 2.50 \\
\hline
\end{tabular}

charged and therefore likely to repel positrons. The present calculations show that already the atomic size effect strongly opposes localization.

The numerical results for positron states in vacancies and divacancies are collected in Table $\mathrm{V}$ and the positron lifetimes in semiconductors are shown also in Fig. 12. The increase of the positron lifetime between the bulk state and the vacancy state in semiconductors is rather small. The ratio of the lifetimes is only about 1.12-1.16. For metals the ratio is typically around 1.5-1.6, reflecting a more localized positron wave function in a close-packed lattice. In the insulators studied the values are near those for metals when a bound state is found. For divacancies the relative increase of the positron lifetime is of the same order as for vacancies in metals. Figure 12 demonstrates that the positron lifetimes corresponding to bulk, single vacancies, and divacancies increase linearly as a function of the unit-cell volume. In the case of vacancies several groups have to be separated according to the size (or the row in the Periodic Table of the elements) of the atom missing. The lifetime simply measures the amount of "open volume" available for the positron.

According to Table $\mathrm{V}$ positron binding energies in vacancies are small, only a few tenths of an eV. This reflects the rather delocalized character of the bound positron state. In a compound system the binding energy is higher in the larger vacancy, i.e., corresponding to the

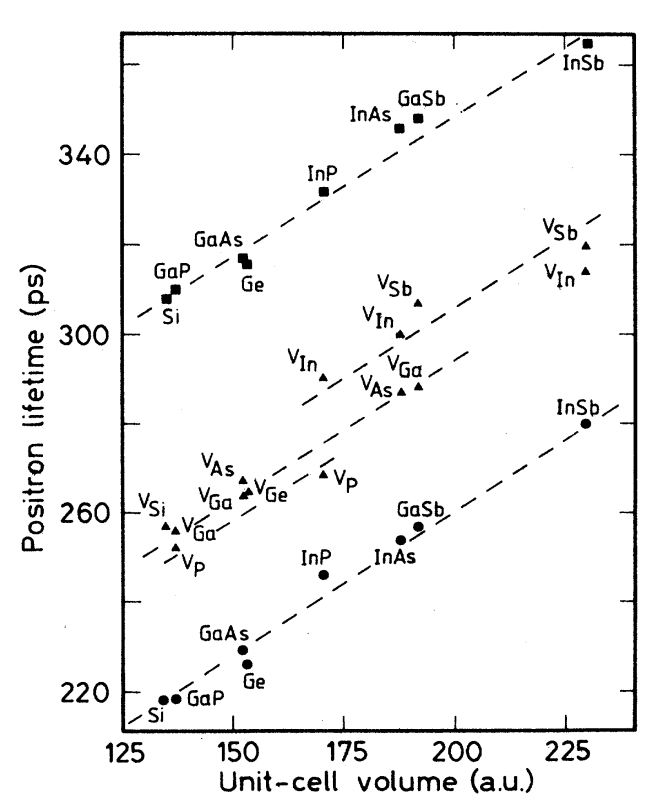

FIG. 12. The calculated lifetimes for positrons delocalized in perfect semiconductor lattices (circles), trapped at vacancies (triangles), and trapped at nearest-neighbor divacancies (squares), as a function of the unit-cell volume. The dashed lines are drawn in order to guide the eye. 
missing atom on the lower row in the Periodic Table, or if the elements are on the same row, the element on the right-hand side.

\section{B. Discussion}

Experimental data for positron lifetimes in vacancies and divacancies exist for $\mathrm{Si}$ and GaAs. For the Si vacancy $\left(V_{\mathrm{Si}}\right)$ the experimental values are $266-270$ ps (Refs. 26 and 27) and for the Si divacancy the values $320-325$ ps (Refs. 26 and 39) have been suggested. The present theoretical predictions are smaller, 256 and 309 ps. This may be due to the omission of the outward lattice relaxation. In the case of GaAs the experimental findings are more complex. The positron lifetime of $260 \mathrm{ps}$ is connected with $V_{\mathrm{Ga}} \cdot{ }^{40}$ For $V_{\mathrm{As}}$ two positron lifetime components of 260 and 295 ps depending on sample conditions have been suggested. ${ }^{6}$ Our theoretical result for $V_{\mathrm{Ga}}$ is $265 \mathrm{ps}$, i.e., slightly above the experimental value. In the case of $V_{\text {As }}$ the experimental results are on both sides of the calculated value of $268 \mathrm{ps}$. According to the model presented by Corbel et $a l^{6}$ the two experimental positron lifetimes correspond to different charge states of the $V_{\text {As. }}$. The change in the positron lifetime can be, at least partly, explained by the change of lattice relaxation. The present model for the screening of positrons in semiconductors opens an additional possibility. Namely, the screening may depend on the charge state of the defect. One obtains an order of magnitude estimate for this effect by using the metallic limit for the screening. In the case of $V_{\text {As }}$ the metallic screening gives a lifetime 12 ps shorter than in the semiconductor model. Dannefaer and $\mathrm{Kerr}^{41}$ have proposed that the lifetime component of 295 ps should be connected with divacancies in GaAs. However, this assignment seems improbable according to present theory, which predicts for the defect a remarkably longer lifetime of 317 ps.

Previously the positron states in vacancies and small vacancy clusters have been calculated by using the Brandt-Reinheimer enhancement model Eq. (14) with $\varepsilon_{g}=0.2 .^{19,22}$ The previous atomic superposition calculations $^{22}$ give essentially the same results as the present calculations. The LMTO-ASA Green's-function calculations, ${ }^{19}$ which use self-consistent electron densities, differ from the present treatment because they can deal with the different charge states. The LMTO-ASA Green'sfunction calculations showed that the positron lifetime in a vacancy does not depend strongly on the charge state if the electron-positron correlation is treated similarly in each case, and no relaxation occurs. The positron lifetimes in vacancies calculated by the LMTO-ASA Green's-function method are similar to the present results. According to the earlier calculations the positron lifetime in $V_{\mathrm{As}}$ is $\sim 10$ ps longer than in the $V_{\mathrm{Ga}}$ whereas the present calculations give only a difference of 3 ps.
This small disagreement reflects the effects of the selfconsistency of the electron density. The positron binding energy in a defect is more sensitive to the details of the electron structure than the lifetime. For example, the LMTO-ASA Green's-function calculations predict for vacancies binding energies which are larger than the present values approximately by a factor of 2 . Moreover, in that scheme the binding energy depends on the charge state of the defect. The actual magnitude of the binding energy has an important effect on the positron trapping rate into the defect. ${ }^{3}$

\section{CONCLUSIONS}

We have developed two models which describe the electron-positron correlation in insulators and semiconductors, in particular the enhancement of the electron density at the positron. The high-frequency dielectric constant has been found to be the relevant quantity for determining the effect of the band gap on the screening response of the valence electrons. The first model, which starts from the properties of electron gas, is better suited to typical semiconductors. This model is free from adjustable parameters and is therefore of great value in supporting the interpretation of measurements, first of all the determination of positron bulk lifetimes. The basis of the second model is to treat the polarizability of the valence electrons as that of more tightly bound core electrons, and the Clausius-Mossotti relation is used. Therefore the model describes better insulators. The model contains two adjustable parameters common for all materials, and it can be considered as an interpolation scheme between different insulator hosts.

We have used the models to predict positron lifetimes at vacancy-type defects in semiconductors and insulators. The main conclusion from the defect calculations is that the lifetime measures faithfully the amount of the open volume available for the positron. This is seen already in the results for the perfect lattices. However, some of the experimental findings are difficult to understand using the present models. Firstly, one should take the lattice relaxation into account at least in the calculation of the electron density for the defect, ${ }^{22}$ but the relaxed positions of the atoms are not accurately known. Secondly, the change of the charge state of the defect affects the relaxation, and it might also affect electron-positron correlation. Thirdly, in the present calculations the average electron density at the defect is not affected by the localized positron as it in principle should be. Calculations performed with the two-component density-functional theory could answer this question.

\section{ACKNOWLEDGMENT}

We are grateful to $P$. Hautojärvi for many helpful discussions.
*Permanent address: Laboratory of Physics, Helsinki University of Technology, SF-02150 Espoo, Finland.

†Permanent address: Department of Physics, University of Jyväskylä, SF-40100 Jyväskylä, Finland.
${ }^{1}$ For reviews, see, e.g., R. M. Nieminen and M. Manninen, in Positrons in Solids, edited by P. Hautojärvi (Springer-Verlag, Heidelberg, 1979); R. M. Nieminen, in Positron Solid State Physics, edited by W. Brandt and A. Dupasquier (North- 
Holland, Amsterdam, 1983).

2J. Arponen and E. Pajanne, Ann. Phys. (N.Y.) 121, 343 (1979); J. Phys. F 9, 2359 (1979).

${ }^{3}$ For a review, see M. J. Puska, Phys. Status Solidi A 102, 11 (1987).

${ }^{4}$ E. Boroński and R. M. Nieminen, Phys. Rev. B 34, 3820 (1986); R. M. Nieminen, E. Boroński, and L. Lantto, ibid. 32, 1377 (1985).

${ }^{5}$ S. Dannefaer, P. Mascher, and D. Kerr, Defects in Electronic Materials, edited by M. Stavola, S. J. Pearton, and G. Davies, Mater. Res. Soc. Symp. Proc. 104, 471 (1988).

${ }^{6}$ C. Corbel, M. Stucky, P. Hautojärvi, K. Saarinen, and P. Moser, Phys. Rev. B 38, 8192 (1988); see also C. Corbel, M. Stucky, P. Hautojärvi, K. Saarinen, and P. Moser, Defects in Electronic Materials, edited by M. Stavola, S. J. Pearton, and G. Davies, Mater. Res. Soc. Symp. Proc. 104, 475 (1988).

${ }^{7}$ G. Dlubek and O. Brümmer, Ann. Phys. (Leipzig) 7, 178 (1986).

${ }^{8}$ G. Dlubek and R. Krause, Phys. Status Solidi A 102, 443 (1987).

${ }^{9}$ S. Dannefaer, Phys. Status Solidi A 102, 481 (1987).

${ }^{10}$ M. J. Puska and R. M. Nieminen, J. Phys. F 13, 333 (1983).

${ }^{11}$ W. Brandt and J. Reinheimer, Phys. Lett. 35A, 109 (1971).

${ }^{12}$ Note that the expression (3) gives slightly smaller values for the enhancement factor than the many-body calculations for homogeneous electron gas in Ref. 2. Moreover, the lowdensity limit in the homogeneous case should in fact be $\mathrm{Ps}^{-}$ ion, where $\lambda=2.0908 \mathrm{~ns}^{-1}$ [Y. K. Ho, J. Phys. B 16, 1503 (1983)]. This is not important in the present context, and for convenience the Brandt-Reinheimer formula (3) is used.

${ }^{13}$ A. E. Carlsson and N. W. Ashcroft, Phys. Rev. B 25, 3474 (1982).

${ }^{14}$ C. H. Leung, M. J. Stott, and C. O. Almbladh, Phys. Lett. 57A, 26 (1976)

${ }^{15}$ E. Bonderup, J. U. Andersen, and D. N. Lowy, Phys. Rev. B 20, 883 (1979).

${ }^{16}$ D. M. Schrader, Phys. Rev. A 20, 918 (1979).

${ }^{17}$ W. Brandt and J. Reinheimer, Phys. Rev. B 2, 3104 (1970).

18J. C. Phillips, Rev. Mod. Phys. 42, 317 (1970).

${ }^{19}$ M. J. Puska, O. Jepsen, O. Gunnarsson, and R. M. Nieminen, Phys. Rev. B 34, 2695 (1986).

${ }^{20}$ T. Chiba, B. Dürr, and W. Brandt, Phys. Status Solidi B 81, 609 (1977).

${ }^{21}$ D. M. Ceperley and B. J. Alder, Phys. Rev. Lett. 45, 566 (1980); we use their local exchange-correlation functional as parametrized by J. Perdew and A. Zunger, Phys. Rev. B 23, 5048 (1981).
${ }^{22}$ M. J. Puska and C. Corbel, Phys. Rev. B 38, 9874 (1988).

${ }^{23}$ N. E. Christensen, S. Satpathy, and Z. Pawlowska, Phys. Rev. B 36, 1032 (1987).

${ }^{24}$ Numerical Data and Functional Relationships in Science and Technology, Vol. 17a of Landolt-Börnstein, edited by $O$. Madelung (Springer-Verlag, Berlin, 1982); R. W. G. Wyckoff, Crystal Structures (Krieger, Malabar, 1982), Vol. 1.

25 J. A. van Vechten, in Handbook of Semiconductors, edited by S. P. Keller (North-Holland, Amsterdam, 1980), Vol. 3; B. R. Nag, Electron Transport in Compound Semiconductors (Springer-Verlag, Heidelberg, 1980).

${ }^{26}$ W. Fuhs, V. Holzhauer, S. Mantl, F. W. Richter, and R. Sturm, Phys. Status Solidi B 89, 69 (1978).

${ }^{27}$ S. Dannefaer, P. Mascher, and D. Kerr, Phys. Rev. Lett. 56, 2195 (1986).

${ }^{28}$ C. Corbel, P. Moser, M. Stucky, Ann. Chim. (Paris) 8, 733 (1985).

${ }^{29}$ G. Dlubek, O. Brümmer, and A. Polity, Appl. Phys. Lett. A 49, 399 (1985).

${ }^{30}$ S. Dannefaer, J. Phys. C 15, 599 (1982).

${ }^{31}$ G. Dlubek, O. Brümmer, F. Plazaola, and P. Hautojärvi, J. Phys. C 19, 331 (1986).

${ }^{32}$ R. Krause (private communication).

${ }^{33}$ C. Corbel (private communication).

${ }^{34}$ G. Dlubek, O. Brümmer, F. Plazaola, P. Hautojärvi, and K. Naukkarinen, Appl. Phys. Lett. 46, 1136 (1985).

${ }^{35}$ A. Sen Gupta, P. Moser, C. Corbel, and P. Hautojärvi, Cryst. Res. Technol. 23, 243 (1988).

${ }^{36}$ B. Geffroy, C. Corbel, M. Stucky, R. Triboulet, P. Hautojärvi, F. L. Plazaola, K. Saarinen, H. Rajainmäki, J. Aaltonen, P. Moser, A. Sengupta, and J. L. Pautrat, in Defects in Semiconductors, edited by H. J. von Bardeleben, Materials Science Forum (Trans Tech Publications, Aedermannsdorff, 1986), Vols. 10-12, p. 1241.

${ }^{37}$ R. Pareja, M. A. Pedrosa, and R. Gonzáles, in Positron Annihilation, edited by P. C. Jain, R. M. Singru, and K. P. Gopinathan (World Scientific, Singapore, 1985), p. 708.

${ }^{38} \mathrm{O}$. K. Andersen, O. Jepsen, and D. Glötzel, in Highlights of Condensed-Matter Theory, edited by F. Bassani, F. Fumi, and M. P. Tosi (North-Holland, Amsterdam, 1985); H. C. Skriver The LMTO Method (Springer, New York, 1984).

${ }^{39}$ S. Dannefaer, G. W. Dean, D. P. Kerr, and B. G. Hogg, Phys. Rev. B 14, 2709 (1976).

${ }^{40}$ P. Hautojärvi, P. Moser, M. Stucky, and C. Corbel, Appl. Phys. Lett. 48, 809 (1986).

${ }^{41}$ S. Dannefaer and D. Kerr, J. Appl. Phys. 60, 591 (1986). 